\title{
Segmentation of Magnetic Resonance Images Using 3D Deformable Models
}

\author{
Jyrki Lötjönen ${ }^{1,2,3}$, Isabelle E. Magnin ${ }^{1}$, Pierre-Jean Reissman ${ }^{1,2}$, Jukka \\ Nenonen $^{2,3}$, and Toivo Katila ${ }^{2,3}$ \\ 1 Creatis, INSA 502, 69621 Villeurbanne Cedex, France \\ Isabelle. Magninøcreatis . insa-lyon . fr \\ ${ }^{2}$ Laboratory of Biomedical Engineering, Helsinki University of Technology, P.O.B. \\ 2200, FIN-02015 HUT, Finland \\ \{Jyrki.Lotjonen, Jukka.Nenonen, Toivo.Katila\}@hut.fi \\ 3 BioMag Laboratory, Helsinki University Central Hospital, P.O.B. 503, FIN-00029 \\ HYKS, Finland
}

\begin{abstract}
A new method to segment MR volumes has been developed. The method matches elastically a 3D deformable prior model, describing the structures of interest, to the MR volume of a patient. The deformation is done using a deformation grid. Oriented distance maps are utilized to guide the deformation process. Two alternative restrictions are used to preserve the geometrical prior knowledge of the model. The method is applied to extract the body, the lungs and the heart. The segmentation is needed to build individualized boundary element models for bioelectromagnetic inverse problem. The method is fast, automatic and accurate. Good results have been achieved for four MR volumes tested so far.
\end{abstract}

\section{Introduction}

Multichannel magneto- and electrocardiographic (MCG and ECG) recordings have been proved useful in non-invasive localization of bioelectromagnetic sources such as cardiac excitation sources [1]. In the bioelectromagnetic forward and inverse problems, the anatomy of the patient has to be modeled as a volume conductor. Usually the modeling is done using magnetic resonance (MR) images of the patient [2]. However, the segmentation of the structures of interest remains in practice the most time consuming part in the modeling process, thus drastically limiting the use of the individualized models.

In medical imaging field, accurate delineation of anatomic structures from image data sequences is still an open problem. In practice, manual extraction of the objects of interest is often considered as the most reliable technique. However, such methods remain time consuming and are affected by intra- and interobserver variability. Various computer methods have been recently proposed for segmentation of medical images [3,4]. They can be divided into region-based and boundary based approaches [5]. In region based methods, some features based on the intensity of the images are used to merge voxels. One of the limitations of 
these approaches is the difficulty to automatically choose seed points. To overcome the problem, higher control can be used with graph theory approaches and prior knowledge of the image content $[6,7]$. The boundary based methods $[8$, 9] rely on an intensity gradient detection. Unfortunately, they are sensitive to impulsive noise, which cause spurious and partial edge maps. Deformable methods $[9,10]$ allow to group partial edges with a chosen parametrization [11]. Their main drawback is the lack of control on the resulting shape [12]. This is especially true when the images contain several nested structures of interest [13]. To deal with the problem, we propose an automatic segmentation technique, based on the deformable pyramidal model [14] that highly relies on the prior geometrical knowledge $[15,16]$ of the anatomic structures to be segmented. The theory of the method is represented more detailed in a recent doctorate thesis [17].

\section{Methods}

The aim of this work is to extract the body surface, lungs and heart from the MRimages as automatically and fast as possible starting from a $3 \mathrm{D}$ prior geometrical deformable model. The model used is a triangulation representing the surfaces of the structures of interest (Fig. 1).

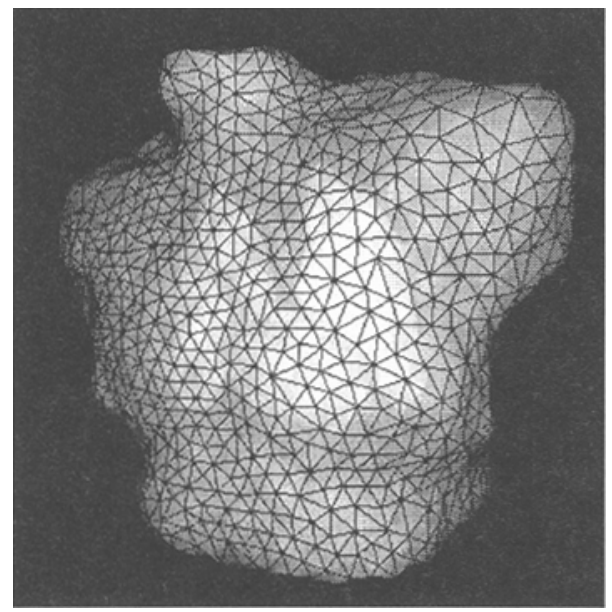

Fig. 1. The prior geometrical model used in the $3 D$ deformation process. It has been built from MR-images of a female patient.

\subsection{The energy function}

1. Isotropic distance maps. The model is elastically deformed in 3D to match to edges in MR images. The edges can be extracted from MR-images using a classical edge detection algorithm [18]. In practice, thresholding separates body surface and lungs from MR-images well enough and the edges can be easily found from the binarized volume. 
Once the edges have been extracted from the volume, the energy function is defined by calculating a distance map $f=f(x, y, z)$ for the edges (Fig. 2) [19]. Each voxel in the distance map gives the shortest chamfer distance to the nearest edge in the 3D MR volume. This leads to so called isotropic distance map. The mismatch energy $E_{\text {data }}$ between the edges in the MR volume and the triangulated prior model is calculated as follows:

$$
E_{\text {data }}=\frac{1}{N_{n d}} \sum_{l=1}^{N_{n d}} f\left(x_{n d, l}, y_{n d, l}, z_{n d, l}\right),
$$

where $N_{n d}$ is the total number of nodes in the model and $x_{n d, l}, y_{n d, l}, z_{n d, l}$ are the coordinates of the node $l$.

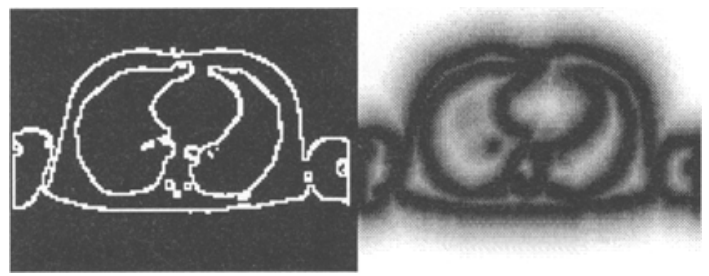

Fig. 2. Calculation of the distance map: a) the edges and b) the corresponding distance map.

2. Oriented distance maps. The prior knowledge about the objects to be segmented can be utilized in the creation of the distance maps; so called oriented distance maps are defined. The idea is that the boundaries of given orientation in the model have to match with similarly oriented edges extracted from the MR-data. In other words, if the surface normal of the model is locally oriented towards the positive $\mathrm{y}$-axis, this part of the surface is allowed to deform only towards the edges in MR data with the same normal directions. To accomplish this, oriented edges are searched from the MR data (Fig. 3). In the middle of Fig. 3, all edges found from a MR-slice are represented. The arrows on the surrounding subimages give the average normal direction of the edges, $\mathbf{n}_{m r, i}$ on each subimage. The normals of the edges within a subimage differ less than $90^{\circ}$ from the vector $\mathbf{n}_{m r, i}$. The number of different orientations is 8 in $2 \mathrm{D}$ (9 neighborhood) and 26 in 3D (27 neighborhood). The normal directions of the edges are estimated from the thresholded MR volume as follows: 1) Calculate the center of mass for each edge point using 27 voxel neighborhood. 2) The normal of the voxel is the vector from the center of mass to the edge point.

Actually all 26 oriented distance maps are not calculated on the highest resolution level for two reasons: 1 ) The size of the distance maps would be tens of megabytes. 2) The execution time would be about 2-3 minutes longer. The problem is solved by calculating only 6 oriented distance maps. The vectors $\mathbf{n}_{m r, i}$ are along x-, y- and z-axis. Since the angle between $\mathbf{n}_{m r, i}$ and the normal of each edge point in an oriented distance map is less than $90^{\circ}$, Eq. 3 gives a 
good distance map for any $\mathbf{n}_{n d, l}$. If the angle is 90 degrees, in practice, 6 distance maps can be used instead of 26 in each resolution level.

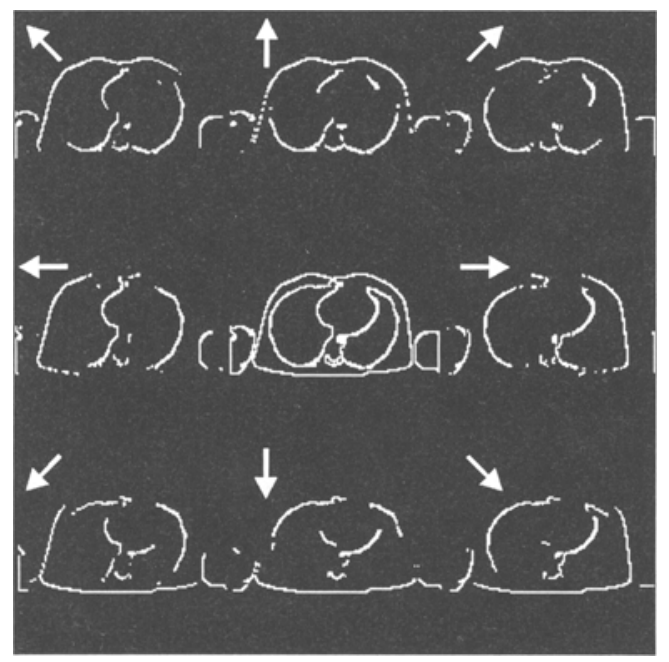

Fig. 3. Oriented edges. The middle image shows all edges extracted from one $M R$ slice. The edges oriented to 8 directions are presented in the surrounding subimages.

The oriented distance maps are created by calculating distance maps separately for these 26 volumes including the oriented edges. The energy of the model can be calculated as follows

$$
E_{d a t a}=\frac{1}{N_{n d}} \sum_{l=1}^{N_{n d}} f_{i}\left(x_{n d, l}, y_{n d, l}, z_{n d, l}\right),
$$

where

$$
f_{i}, i \in\{1, . ., 26\}: \mathbf{n}_{m r, i} \cdot \mathbf{n}_{n d, l} \geq \mathbf{n}_{m r, k} \cdot \mathbf{n}_{n d, l}, \forall k \in\{1, . ., 26\} .
$$

Here, the vector $\mathbf{n}_{m r, i}$ is the mean orientation of the edges in the distance map $f_{i}$, the vector $\mathbf{n}_{n d, l}$ is the normal of the surface of the model at the node $l$ and . is for dot product. The matching process is now performed in two steps.

\subsection{Coarse matching}

The model is coarsely registrated with the MR volume. Surface registration methods, such as [21], can be used to registrate the model with the edges extracted from the MR volume. If the rotation component is small, the bounding box, set around the model, can be scaled in such a way that it coincides with the bounding box set around the thresholded volume. 


\subsection{Detailed matching}

The final registration step is done by placing the model inside a $3 \mathrm{D}$ deformation grid $G$ (Fig. 4). When a grid point is moved, the model is deformed correspondingly. The benefit in using a deformation grid is, that the topology of the model can be easily preserved during the deformation process. The grid points are not moved more than half the distance between two neighboring grid points. The Bernstein polynomials or trilinear interpolation can be used to calculate the new positions for the nodes of the model [22]. In this paper trilinear interpolation is used. The grid divides space into box shaped subvolumes and each node of the triangulated model belongs to one of these boxes. The new position of a node in the model can be calculated as follows

$$
\mathbf{p}_{n d}=\mathbf{p}_{n d}^{*}+\sum_{k=n}^{n+1} \sum_{j=m}^{m+1} \sum_{i=l}^{l+1} a_{G_{i j k}} \mathbf{v}_{G_{i j k}},
$$

where $\mathbf{p}_{n d}^{*}$ is the original position of the node represented in the vector form, the summing is through the grid points, which define the box around the node, $\mathbf{v}_{G_{i j k}}$ is the displacement vector of the grid point $G_{i j k}$ from its original position and $a_{G_{i j k}}$ is a weighting factor. The factor is calculated using trilinear interpolation.

All grid points $G_{i j k}$ are sequentially moved towards a new position, which minimizes the energy of the model. This energy is computed from the distance map as defined in Eq. 2. within a $3 \times 3 \times 3$ or $5 \times 5 \times 5$ voxel neighborhood. Each grid point $G_{i j k}$ is moved within the neighborhood and displacement is done sequentially for every point in the grid $G$. The process is iterated until the energy does not decrease anymore.

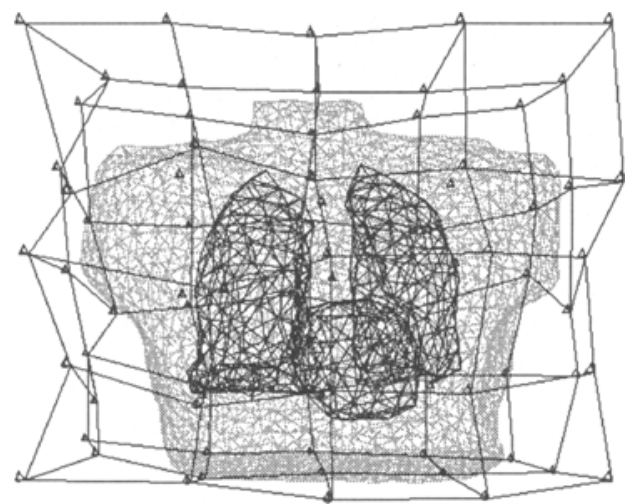

Fig. 4. The 5x3x5 deformation grid $G$ and the model placed inside.

The deformation can be started for example by a $3 \times 3 \times 3$ grid, so providing a global match between the model and the MR volume. When the minimum is found for a given grid size, the number of grid points can be increased to $5 \times 5 \times 5$, 
$7 \times 7 \times 7$ etc. Initial positions of the points in the new grid are regularly placed and not interpolated from the earlier, deformed grid.

To ensure a theoretical convergence towards the global minimum, methods such as simulated annealing should be used [20]. Since these methods are very time consuming, we chose the pyramid approach [21]. It does not guarantee the global minimum but works better than the method using local gradient alone.

In the case where the extraction of the edges from MR data does not succeed, some false edges may appear or some true edges may remain undetected. If the minimum of energy is defined using only gradient information, the deformation can lead to unacceptable results. In that case restrictions for deformation strength should be set. Reissman [17] proposed to limit the displacements of the grid points (LGD=Limit Grid Displacements) and proposed the deformation component of the energy to be calculated as follows,

$$
E_{\text {model }}=\frac{1}{N_{G}} \sum_{l=1}^{N_{G}}\left\|\mathbf{p}_{G, l}-\mathbf{p}_{G, l}^{*}\right\|,
$$

where $N_{G}$ is the number of grid points, $\mathbf{p}_{G, l}$ is the deformed position of the grid point $l$ and $\mathbf{p}_{G, l}^{*}$ its original position.

Another way to preserve the prior knowledge of the model during deformation is to directly control the changes in the shape of the model. If the directions of the surface normals are used (LCN = Limit Change of Normals), the deformation energy $E_{\text {model }}$ can be calculated as follows

$$
E_{\text {model }}=\frac{1}{N_{t r}} \sum_{l=1}^{N_{t r}}\left(1.0-\mathbf{n}_{t r, l} \cdot \mathbf{n}_{t r, l}^{*}\right),
$$

where $N_{t r}$ is the total number of triangles in the model, $\mathbf{n}_{t r, l}$ and $\mathbf{n}_{t r, l}^{*}$ are the deformed and the original directions of the normal of the triangle $l$, respectively, and $\cdot$ is the dot product. Since $\mathbf{n}_{\mathbf{t r}, \mathbf{l}} \cdot \mathbf{n}_{t r, l}^{*}=\cos \left(\Varangle\left(\mathbf{n}_{t r, l}, \mathbf{n}_{t r, l}^{*}\right)\right)$, the large changes in the normal direction penalize more deeply to the energy than small changes.

Using deformation component in the energy, the total energy is defined by

$$
E_{\text {total }}=E_{\text {data }}+\gamma E_{\text {model }}
$$

where $\gamma$ is a user defined parameter to control the balance between the data feature and the deformation components.

\section{Results}

The method was tested to segment the thorax, lungs and heart of four patients from their MR volumes using an IBM RS6000 workstation. In each case the algorithm produced a good result, with few interactive corrections of small magnitude. Next, the results for one thorax MR are represented. The size of the MR volume is $128 \times 128 \times 100$. Fig. 5 shows the result of prior model deformation, when 




Fig. 5. Segmentation result with $\gamma=0.0$, with the LGD-method $(\gamma=0.01)$ and with the $L C N$-method $(\gamma=5.0)$

$\gamma=0.0$, when the LGD-method was used with $\gamma=0.01$ and when the LCNmethod was applied with $\gamma=5$. The results are not very sensitive to $\gamma$-values in the LGD- and LCN-methods: increase by factor 10 produces fairly similar results. The numbers of grid points on each pyramid level are represented in Table 1a. The iteration with each grid size was stopped, when the change in the energy was less than user defined parameter $\epsilon=0.02$. The computing time to segment MR images and the energies at convergence related to the Fig. 5 are represented in Table $1 \mathrm{~b}$. The star ' $*$ ' indicates that the oriented distance maps are not used at the highest resolution level, other parameters being equal. $E_{d a t a}$ corresponds to the average distance (in $\mathrm{mm}$ ) between the nodes of the model and the edges in the MR volume. Since the voxel size at the highest resolution is $3.52 \mathrm{~mm}$, the average distance of the nodes from edges is 0.17 voxel.

\section{Discussion}

A. General discussion. The segmentation error with the automatic method is low: about $87 \%$ of the nodes of the model are located on the edges of the MR data and the average error is about 0.17 voxel for the case presented in the

\begin{tabular}{|c|c|c|c|}
\hline Level & Resolution & Grid & Model \\
\hline 2 & $32 \times 32 \times 25$ & $3 \times 3 \times 3,5 \times 5 \times 5$ & 983 \\
\hline 1 & $64 \times 64 \times 50$ & $5 \times 5 \times 5,5 \times 5 \times 5$ & 2713 \\
\hline 0 & $128 \times 128 \times 100$ & $\begin{array}{c}7 \times 7 \times 7,9 \times 9 \times 9 \\
11 \times 11 \times 11\end{array}$ & 9811 \\
\hline
\end{tabular}

\begin{tabular}{|l|c|c|c|c|}
\hline Method & Time & Edata & Emodel & Etotal \\
\hline$\gamma=0.0$ & 156 & 0.56 & 0.00 & 0.56 \\
LGD & 155 & 0.58 & 0.11 & 0.69 \\
LCN & 211 & 0.61 & 0.10 & 0.71 \\
\hline$\gamma=0.0^{*}$ & 94 & 0.61 & 0.00 & 0.61 \\
LGD $^{*}$ & 95 & 0.59 & 0.11 & 0.80 \\
LCN $^{*}$ & 150 & 0.62 & 0.11 & 0.73 \\
\hline
\end{tabular}

Table 1. a) The sizes of the grid and the number of nodes in the model at the different pyramid levels. b) Computing time (seconds) and matching energy for each method. 
results section. This error can be still reduced by increasing the size of the deformation grid. It is remarkable that, when only the $E_{\text {data }}$ component is minimized $(\gamma=0.0)$, the energy value is higher than in the case of the LGD-method. This means the algorithm does not find the global minimum. However, in most cases the results are 'good' local minima. The segmentation results are also visually accurate, which is even more important than the absolute value of the error. The absolute value should not be emphasized too much, since the error can be low, although the model has deformed to wrong edges.

The model may also include edges, which can not be found from the data. For example, for the thorax three areas exist: 1) The model used in our MCG/ECG studies does not include arms (Fig. 1). 2) The edges of the heart can not be easily found in regions where the heart is not in contact with the lungs. If these areas are deformed to some edges in the MR volume data, the result is wrong, although the $E_{\text {total }}$ can be minimum. 3) The quality of the MR images is poor nearby the shoulders, and the edges extracted from the volume does not describe well the real anatomy. In these cases, it is almost compulsory to use $E_{\text {model }}$ component, which preserves the geometrical prior knowledge.

The balance between the $E_{d a t a}$ and $E_{\text {model }}$ components can be set locally to improve the handling of the mismatch problem of the model and real data. This can be accomplished, for example, by attaching a weight $t_{n d, l}$ to each node of the model and multiplying the $E_{\text {data }}$ term of the corresponding node by this weight.

In this paper two different methods to define $E_{\text {model }}$ were described: the LGDand the LCN-methods. The biggest benefit of the LGD-method is its speed. The quality of the results did not differ a lot for the cases we tested. However, the LCN-method preserves better the geometrical prior knowledge.

$B$. Implementation. Several factors affect to the execution time. If the oriented distance maps are used, the running time is higher (Table 1) but the robustness of the program is better. However, the results are fairly similar to the results with the oriented distance maps. The optimization time is directly dependent on the number of the nodes in the model. However, the number of the nodes can not be reduced too much. Otherwise, the probability that the model attaches to noise increases considerably. The average distance between the nodes was about two voxels at each resolution level. The size of the deformation grid does not affect much the execution time. If the size is higher, the number of the nodes connected to each grid point is correspondingly lower. Each grid size affects the run time cumulatively. For example, if a $11 \times 11 \times 11$ grid had not been used in Table 1, the time would have been $10 \mathrm{~s}$ lower. The average error of the deformation would have been $0.81 \mathrm{~mm}$, not $0.56 \mathrm{~mm}$.

In summary, a new method to segment MR volumes has been developed. Since the method is fast and the results have been good for all tested data, it can be regarded useful in practice. 


\section{References}

1. Nenonen, J.: Solving the inverse problem in magnetocardiography. IEEE Eng. Med. Biol. 13 (1994) 487-496

2. Lötjönen, J., Reissman, P-J., Magnin, I.E., Nenonen, J. and Katila, T.: A Triangulation Method of an Arbitrary Point Set for Biomagnetic Problems. IEEE Trans. Magn. in press

3. Amit, Y., Kong, A.: Graphical templates for model registration. IEEE Trans. PAMI 18 (1996) 225-236

4. Philip, K.P., Dove, E.L., McPherson, D., Gotteiner, N.L., Vonesh, M.J., Stanford, W., Reed, J.E., Rumberger, J.A., Chanbran, K.B.: Automatic detection of myocardial contours in cine computed tomographic images. IEEE Trans. Med. Imag. 13 (1994) 241-253

5. Worring, M.W., Smeulders, A.W.M., Staib, L.H., Duncan, J.S.: Parameterized feasible boundaries in gradient vector fields. Comput. Vision Imag. Under. 63 (1996) 135-144

6. Fwu, J.K., Djurie, P.M.: Unsupervised vector image segmentation by a tree structure-ICM algorithm. IEEE Trans. Med. Imag. 15 (1996) 871-880

7. Sonka, M., Tadikonda, S.K., Collins, S.M.: Knowledge-based interpretation of MR brain images. IEEE Trans. Med. Imaging 44 (1996) 443-452

8. Goshtasby, A., Turner, D.A.: Segmentation of cardiac cine MR images for extraction of right and left ventricular chambers. IEEE Trans. Med. Imag. 14 (1995) 56-64

9. Chakrabotry, A., Staib, L.H., Duncan, J.S.: Deformable boundary finding in medical images by integrating gradient and region information. IEEE Trans. Med. Imag. 15 (1996) 859-870

10. Davatzikos, C.A., Bryan, R.N.: Using a deformable surface model to obtain a shape representation of the cortex. IEEE Trans. Med. Imag. 15 (1996) 785-795

11. Cohen, L.D., Cohen, I.: Finite-element methods for active contour models and balloons for 2-D and 3-D images. IEEE Trans. PAMI 15 (1993) 1131-1147

12. Kass, M., Witkin, A., Terzopoulos, D.: Snakes: active contour models. Inter. Journ. Comp. Vision 1 (1988) 321-331

13. Davatzikos, C.A., Prince, J.L.: An active contour model for mapping the cortex. IEEE Trans. Med. Imag. 14 (1995) 65-80

14. Reissman, P-J., Magnin, I.E.: Modeling 3D deformable object with the active pyramid. Int. J. Patt. Rec. \& Art. Int. 11 (1997) 1129-1139

15. Jain, A.K., Zhong, Y., Lakshmannan, S.: Object matching using deformable templates. IEEE Trans. PAMI 14 (1996) 267-278

16. Terzopoulos, D., Waters, K.: Analysis and synthesis of facial image sequences using physical and anatomical models. IEEE Trans. Med. Imag. 15 (1993) 569-579

17. Reissman, P-J.: Modélisation et mise en correspondance par pyramides actives: Application à l'imagerie cardique par résonance magnétique, Doctorate thesis, L'Institut National Des Sciences Appliquees de Lyon France (1997)

18. Canny, J.: A computational approach to edge detection. IEEE Trans. PAMI 8 (1986) 679-698

19. Borgefors, G.: Distance transformation in digital images. Computer Vision Graphics and Image Processing 48 (1986) 344-371

20. Geman, S., Geman, D.: Stochastic relaxation, Gibbs distributions, and the Bayesian restoration of image. IEEE PAMI 6 (1984) 721-741

21. Borgefors, G.: Hierarchical chamfer matching: A parametric edge matching algorithm. IEEE Trans. PAMI 10 (1988) 849-865

22. Sederberg, T., Parry, S.: Free-form deformation of solid geometrical models. SIGGRAPH 20 (1986) 151-160 